\title{
Towards a mathematical foundation of minimum-variance theory
}

\author{
Jianfeng Feng ${ }^{1}$, Kewei Zhang ${ }^{2}$ and Gang Wei ${ }^{3}$ \\ ${ }^{1}$ COGS, Sussex University, Brighton BN1 9QH, UK \\ 2 SMS, Sussex University, Brighton BN1 9QH, UK \\ ${ }^{3}$ Mathematical Department, Baptist University, Hong Kong, People's Republic of China
}

Received 11 January 2002, in final form 18 June 2002

Published 15 August 2002

Online at stacks.iop.org/JPhysA/35/7287

\begin{abstract}
The minimum-variance theory which accounts for arm and eye movements with noise signal inputs was proposed by Harris and Wolpert (1998 Nature 394 780-4). Here we present a detailed theoretical analysis of the theory and analytical solutions of the theory are obtained. Furthermore, we propose a new version of the minimum-variance theory, which is more realistic for a biological system. For the new version we show numerically that the variance is considerably reduced.
\end{abstract}

PACS numbers: 87.19.La, 02.30.Yy, 02.50.Ey

\section{Introduction}

One of the most puzzling issues in neuroscience is why neurons employ stochastic rather than deterministic signals to process information (for example, see reviews $[1,2,4,5,8,13,20]$ ). There are many different approaches to tackle the problem such as stochastic resonance [7], correlated computation [18] and others [9, 10]. Recently Harris and Wolpert [11] (also see recent review [23]) proposed a theory, the minimum-variance theory, as an example of optimal control models of movement, aiming to provide a plausible explanation of 'noise calculation' in the cortex. The idea of the theory is quite straightforward: to find a noise signal which minimizes the variance of certain quantities, say the arm movement trajectory.

For concreteness, let us first formulate their theory. The discrete time version used in $[11,19]$ is

$$
\boldsymbol{x}_{t+1}=A \boldsymbol{x}_{t}+C\left(u_{t}+k u_{t}^{\alpha} \xi_{t}\right)
$$

where $\boldsymbol{x}_{0}=\mathbf{0}\left(\in \mathbb{R}^{d}\right), A$ is a $d \times d$ matrix, $C$ is a $d \times 1$ matrix, $k, \alpha$ are positive constants ${ }^{4}$, $\xi_{t}$ is a normal random variable and $u(t) \in \mathbb{R}$ is the input signal. $\boldsymbol{x}(t) \in \mathbb{R}^{d}$ could be the movement trajectory, velocity, etc. More precisely, the term

$$
u_{t}+k u_{t}^{\alpha} \xi_{t}
$$

${ }^{4} \alpha=1$ in [11], see also [6]. 
is the input signal. As we all know, it is generally agreed that the output of a neuron usually takes the form of a Poisson process (see the discussion). For a Poisson process, it can be approximated by equation (1.2) (see page $166[21]$ ) and $u_{t}$ is the rate of the Poisson process. Note that equation (1.2) is fully determined by $u_{t}$. Without introducing ambiguity, when we speak about an input signal, we could mean either $u_{t}$ or equation (1.2). Analytically it is not easy to deal with a discrete time system, so we rewrite equation (1.1) as the following continuous time version:

$$
\left\{\begin{array}{l}
\mathrm{d} \boldsymbol{x}(t)=(A-I) \boldsymbol{x}(t) \mathrm{d} t+\boldsymbol{C}\left(u(t) \mathrm{d} t+k u^{\alpha}(t) \mathrm{d} B_{t}\right) \\
\boldsymbol{x}(0)=\mathbf{0}
\end{array}\right.
$$

where $B_{t}$ is the standard Brownian motion and $I=\left(\delta_{i j}\right)_{d \times d}$. We therefore have

$\boldsymbol{x}(t)=\int_{0}^{t} \exp ((A-I)(t-s)) \boldsymbol{C u}(s) \mathrm{d} s+\int_{0}^{t} \exp ((A-I)(t-s)) C k u^{\alpha}(s) \mathrm{d} B_{s}$.

For a vector $D \in \mathbb{R}^{d}$ it is required that

$$
\boldsymbol{E} \boldsymbol{x}(T)=\int_{0}^{T} \exp ((A-I)(T-s)) \boldsymbol{C u}(s) \mathrm{d} s=\boldsymbol{D} \in \mathbb{R}^{\mathrm{d}}
$$

i.e., at time $T$ the mean trajectory ends at the position $D$. Equation (1.4) is the constraint for all problems considered below.

In [11] it is assumed that the variance of $\boldsymbol{x}(t)$ in time window $[T, T+R]$ is minimized, i.e. post-movement minimization of variance. More specifically, their minimum-variance theory can be summarized as

- To find an input signal $u(t), t \in[0, T+R]$ such that the generalized total variance of $\boldsymbol{x}(t)$ is minimized for $t \in[T, T+R], R>0$.

Harris and Wolpert [11] compared their simulation results with experimental data and concluded that their results based upon the minimum-variance theory (MVT) fit well with arm and eye movements. Recently the MVT has become quite influential. It leads to many interesting open problems for further theoretical and experimental investigations. The corresponding results might provide a clue for 'noise calculation'.

Despite the success of the MVT model, there are quite a few issues which remain elusive. For example, Feng et al [6] pointed out that the signal used in the minimum-variance theory is not a (limit) Poisson process, in contrast with the claim in [19]. Furthermore, how the model behaviour depends on specific parameters used in the simulations is not clear. Here we first develop a theoretical approach to the minimum-variance theory with post-movement minimization. Some analytical solutions of the theory are obtained. Unfortunately, at the moment it is impossible to directly compare our theoretical solutions with their numerical results in $[11,22]$.

The theory of minimizing the variance of post movement is interesting, but quite frequently a biological system might care more about its behaviour before rather than after its motion has been stopped. The simplest analogue is a person passing a glass of milk to another person but without spilling a drop. Thus, we propose a new version of the minimum-variance theory: minimizing the variance during movement rather than after it. In other words, we intend to minimize the variance during time window $[T-R, T]$ for $T \geqslant R>0$. Again in this case, analytical solutions are obtained. Numerically, as one would expect, we find that the larger the $R$ is, the smaller the variance.

Mathematically, the problem we discuss here is a specific one from the stochastic control theory. Usually to find the optimal control is equivalent to finding the solution of the HamiltonJacobi-Bellman (HJB) equation [14]. Unfortunately, to solve the HJB equation is again a 
formidable task and various numerical methods are proposed. Here, since the time $T$ is deterministic and the control problem is reduced to the open loop control problem ([16] p 149, remark 1), we are able to find the analytical solutions of the optimal control problem. In the following, we always assume that $u$ is in $L^{2 \alpha}[0, T+R]$.

\section{MVT of post movement}

2.1. Minimizing the generalized variance of $\left(\int_{T}^{T+R} \boldsymbol{x}(s) \mathrm{d} s\right)$

Similar to [11], we consider the procedure to minimize the generalized variance of $\left(\int_{T}^{T+R} \boldsymbol{x}(s) \mathrm{d} s\right)$, i.e., the trace of $\operatorname{Cov}\left(\int_{T}^{T+R} \boldsymbol{x}(s) \mathrm{d} s\right)$. For $R>0$, let us define

$$
\begin{array}{r}
\boldsymbol{Y}=\int_{T}^{T+R} \boldsymbol{x}(t) \mathrm{d} t=\int_{T}^{T+R} \int_{0}^{t} \exp ((A-I)(t-s)) C u(s) \mathrm{d} s \mathrm{~d} t \\
+\int_{T}^{T+R} \int_{0}^{t} \exp ((A-I)(t-s)) C k u^{\alpha}(s) \mathrm{d} B_{s} \mathrm{~d} t .
\end{array}
$$

Hence $\boldsymbol{Y}$ is the average trajectory over time window $[T, T+R]$. Remember that the trajectory stops at time $T$; therefore, in time interval $[T, T+R]$ the movement has stopped (post movement).

We first calculate the generalized variance of $\boldsymbol{Y}$.

$$
\begin{aligned}
& \mathbf{E}\left(\boldsymbol{Y}^{\prime} \boldsymbol{Y}\right)=\left\|\int_{T}^{T+R} \int_{0}^{t} \exp ((A-I)(t-s)) \boldsymbol{C} u(s) \mathrm{d} s \mathrm{~d} t\right\|^{2} \\
&+ 2 \mathbf{E}\left\langle\int_{T}^{T+R} \int_{0}^{t} \exp ((A-I)(t-s)) \boldsymbol{C} u(s) \mathrm{d} s \mathrm{~d} t,\right. \\
&\left.\int_{T}^{T+R} \int_{0}^{t} \exp ((A-I)(t-s)) C k u^{\alpha}(s) \mathrm{d} B_{s} \mathrm{~d} t\right\rangle \\
&+\mathbf{E}\left\|\int_{T}^{T+R} \int_{0}^{t} \exp ((A-I)(t-s)) C k u^{\alpha}(s) \mathrm{d} B_{s} \mathrm{~d} t\right\|^{2} \\
&=\left\|\int_{T}^{T+R} \int_{0}^{t} \exp ((A-I)(t-s)) C u(s) \mathrm{d} s \mathrm{~d} t\right\|^{2} \\
&+\mathbf{E}\left\|\int_{T}^{T+R} \int_{0}^{t} \exp ((A-I)(t-s)) C k u^{\alpha}(s) \mathrm{d} B_{s} \mathrm{~d} t\right\|^{2}
\end{aligned}
$$

where $\langle\cdot, \cdot\rangle$ means inner product and we used the fact that

$$
\mathbf{E} \int_{T}^{T+R} \int_{0}^{t} \exp ((A-I)(t-s)) C k u^{\alpha}(s) \mathrm{d} B_{s} \mathrm{~d} t=0
$$

Lemma 1. For $R>0$, we have

$$
\begin{aligned}
\mathbf{E}\left\|\int_{T}^{T+R} \int_{0}^{t} \exp ((A-I)(t-s)) C k u^{\alpha}(s) \mathrm{d} B_{s} \mathrm{~d} t\right\|^{2} \\
\quad=2 \int_{T}^{T+R}(T+R-t) \int_{0}^{t}\|\exp ((A-I)(t-s)) C\|^{2} k^{2} u^{2 \alpha}(s) \mathrm{d} s \mathrm{~d} t .
\end{aligned}
$$


Proof. Recall that ([15], chapter 15)

$$
\begin{aligned}
\int_{T}^{T+R} \int_{0}^{t} \exp ((A-I)(t-s)) C k u^{\alpha}(s) \mathrm{d} B_{s} \mathrm{~d} t \\
=\lim _{h \rightarrow 0} \sum_{i=1}^{n} h \int_{0}^{t_{i}} \exp ((A-I)(t-s)) C k u^{\alpha}(s) \mathrm{d} B_{s}
\end{aligned}
$$

where $T=t_{1}, T+R=t_{n}$ and $t_{i}=t_{i-1}+h$ for $i=2, \ldots, n$ and lim is in $L^{2}$ sense. We then have

$$
\begin{aligned}
\mathbf{E}\left\|\int_{T}^{T+R} \int_{0}^{t} \exp ((A-I)(t-s)) C k u^{\alpha}(s) \mathrm{d} B_{s} \mathrm{~d} t\right\|^{2} \\
=\lim _{h \rightarrow 0} \mathbf{E} \sum_{i, j=1}^{n} h^{2}\left\langle\int_{0}^{t_{i}} \exp ((A-I)(t-s)) C k u^{\alpha}(s) \mathrm{d} B_{s},\right. \\
\\
\left.\quad \int_{0}^{t_{j}} \exp ((A-I)(t-s)) C k u^{\alpha}(s) \mathrm{d} B_{s}\right\rangle .
\end{aligned}
$$

Note that for $t_{i} \geqslant t_{j}$, the integral

$$
\int_{0}^{t_{i}} \exp ((A-I)(t-s)) C k u^{\alpha}(s) \mathrm{d} B_{s}
$$

is also a $\mathbf{0}$ mean martingale ([15], chapter 15). This leads to

$$
\begin{aligned}
\mathbf{E}\left\langle\int_{0}^{t_{i}} \exp ((A-I)(t-s)) C k u^{\alpha}(s) \mathrm{d} B_{s}, \int_{0}^{t_{j}} \exp ((A-I)(t-s)) C k u^{\alpha}(s) \mathrm{d} B_{s}\right\rangle \\
=\mathbf{E}\left\langle\left[\int_{t_{j}}^{t_{i}} \exp ((A-I)(t-s)) C k u^{\alpha}(s) \mathrm{d} B_{s}+\int_{0}^{t_{j}} \exp ((A-I)\right.\right. \\
\\
\left.\left.\quad \times(t-s)) C k u^{\alpha}(s) \mathrm{d} B_{s}\right] \int_{0}^{t_{j}} \exp ((A-I)(t-s)) C k u^{\alpha}(s) \mathrm{d} B_{s}\right\rangle \\
\left.=\mathbf{E} \| \int_{0}^{t_{j}} \exp ((A-I)(t-s)) C k u^{\alpha}(s) \mathrm{d} B_{s}\right) \|^{2} \\
=\int_{0}^{t_{j}}\|\exp (2(A-I)(t-s)) C\|^{2} k^{2} u^{2 \alpha}(s) \mathrm{d} s .
\end{aligned}
$$

Equation (2.3) then turns out to be

$$
\begin{gathered}
\mathbf{E}\left(\int_{T}^{T+R} \int_{0}^{t} \exp ((A-I)(t-s)) C k u^{\alpha}(s) \mathrm{d} B_{s} \mathrm{~d} t\right)^{2} \\
\quad=\lim _{h \rightarrow 0} \sum_{i, j=1}^{n} h^{2} \int_{0}^{\min \left(t_{i}, t_{j}\right)}\|\exp ((A-I)(t-s)) C\| k^{2} u^{2 \alpha}(s) \mathrm{d} s \\
=2 \int_{T}^{T+R}(T+R-t) \int_{0}^{t}\|\exp ((A-I)(t-s)) C\|^{2} k^{2} u^{2 \alpha}(s) \mathrm{d} s \mathrm{~d} t .
\end{gathered}
$$

Summarizing the above arguments, we have the following theorem.

\section{Theorem 1.}

$\mathbf{E}\|\boldsymbol{Y}\|^{2}-\|\mathbf{E} \boldsymbol{Y}\|^{2}=2 \int_{T}^{T+R}(T+R-t) \int_{0}^{t}\|\exp ((A-I)(t-s)) C\|^{2} k^{2} u^{2 \alpha}(s) \mathrm{d} s \mathrm{~d} t$. 
In terms of theorem 1, the original optimization problem can be stated as to find a function $u^{*}(t)$ such that the functional

$I(u)=\left[2 k^{2} \int_{T}^{T+R}(T+R-t) \int_{0}^{t} \| \exp \left((A-I)(t-s) C \|^{2}|u(s)|^{2 \alpha} \mathrm{d} s \mathrm{~d} t\right]\right.$

is minimized, i.e., $I\left(u^{*}\right)=\min _{u} I(u)$, subject to the constraint

$$
\int_{0}^{T} \exp ((A-I)(T-s)) C u(s) \mathrm{d} s=\boldsymbol{D} \in \mathbb{R}^{d} .
$$

This is an infinite-dimensional optimization problem since we have to find a function in $L^{2 \alpha}[0, T+R]$ satisfying equations (2.5) and (2.6).

Theorem 2. Let $\boldsymbol{F}(t-s)=\exp ((A-I)(t-s)) \boldsymbol{C}$ and $H(s)=\int_{T}^{T+R}(T+R-t)$ $\|\boldsymbol{F}(t-s)\|^{2} \mathrm{~d} t$, for $s \in[0, T]$. Then the unique solution of equations (2.5) and (2.6) $u^{*}(t)$ is given by

$$
u^{*}(s)=\left\{\begin{array}{cl}
\frac{\operatorname{sgn}\left(\boldsymbol{\lambda}^{*} \boldsymbol{F}(T-s)\right)\left\|\boldsymbol{\lambda}^{*} \boldsymbol{F}(T-s)\right\|^{1 /(2 \alpha-1)}}{H^{1 /(2 \alpha-1)}(s)} & t \in[0, T] \\
0 & t \in[T, T+R]
\end{array}\right.
$$

where $\lambda^{*} \in \mathbb{R}^{d}$ minimizes

$$
J(\boldsymbol{\lambda})=\left[\int_{0}^{T} \frac{\|\boldsymbol{\lambda} \boldsymbol{F}(T-s)\|^{2 \alpha /(2 \alpha-1)}}{H^{1 /(2 \alpha-1)}(s)} \mathrm{d} s\right]
$$

i.e., $J\left(\boldsymbol{\lambda}^{*}\right)=\inf _{\boldsymbol{\lambda} \in \mathbb{R}^{d}}(J(\boldsymbol{\lambda}))$, with the constraint

$$
\int_{0}^{T} \boldsymbol{F}(T-s) \frac{\operatorname{sgn}(\boldsymbol{\lambda} \boldsymbol{F}(T-s))\|\boldsymbol{\lambda} \boldsymbol{F}(T-s)\|^{1 /(2 \alpha)}}{H^{1 /(2 \alpha-1)}(s)} \mathrm{d} s=\boldsymbol{D} .
$$

Proof. It is easily seen that

$$
\begin{aligned}
I(u)=2 k^{2} \int_{T}^{T+R}(T+R-t) \int_{0}^{t}\|\boldsymbol{F}(t-s)\|^{2}|u(s)|^{2 \alpha} \mathrm{d} s \mathrm{~d} t \\
=2 k^{2} \int_{0}^{T} \mid u(s)^{2 \alpha}\left(\int_{T}^{T+R}(T+R-t)\|\boldsymbol{F}(t-s)\|^{2} \mathrm{~d} t\right) \mathrm{d} s \\
\quad+2 k^{2} \int_{0}^{T} \mid u(s)^{2 \alpha}\left(\int_{T}^{T+R-s}(T+R-t)\|\boldsymbol{F}(t-s)\|^{2} \mathrm{~d} t\right) \mathrm{d} s \\
=2 k^{2} \int_{0}^{T}|u(s)|^{2 \alpha} H(s) \mathrm{d} s+\int_{T}^{T+R}|u(s)|^{2 \alpha} G(s) \mathrm{d} s
\end{aligned}
$$

where

$$
\begin{array}{ll}
H(s)=\int_{T}^{T+R}(T+R-t)\|\boldsymbol{F}(t-s)\|^{2} \mathrm{~d} t & s \in[0, T] \\
G(s)=\int_{T+R-s}^{T+R}(T+R-t)\|\boldsymbol{F}(t-s)\|^{2} \mathrm{~d} t & s \in[T, T+R] .
\end{array}
$$

Let us define

$$
\left\{u, \int_{0}^{T} \boldsymbol{F}(T-s) u(s) \mathrm{d} s=\boldsymbol{D}\right\}=\mathcal{U}_{D}
$$


For small $\tau$, consider $u+\tau \phi \in \mathcal{U}_{D}$, i.e.

$$
\phi \in\left\{\phi, \int_{0}^{T} \boldsymbol{F}(T-s) \phi(s) \mathrm{d} s=0\right\}=\mathcal{U}_{D}^{0}
$$

Since $I(u)$ is strictly convex and coercive, there is a unique minimizer in $L^{2 \alpha}[0, T+R]$ and $u \in \mathcal{U}_{D}$. We then have

$$
\left.\frac{\mathrm{d} I(u+\tau \phi)}{\mathrm{d} \tau}\right|_{\tau=0}=0
$$

which gives

$\int_{0}^{T}|u(s)|^{2 \alpha-1} \operatorname{sgn}(u(s)) \phi(s) H(s) \mathrm{d} s+\int_{T}^{T+R}|u(s)|^{2 \alpha-1} \operatorname{sgn}(u(s)) \phi(s) G(s) \mathrm{d} s=0$.

Since there is no restriction for $\phi(s)$ for $s \in[T, T+R]$ except that $\phi \in L^{2 \alpha}[T, T+R]$, one obtains

$$
|u(s)|^{2 \alpha-1} \operatorname{sgn}(u(s)) \phi(s) G(s)=0
$$

a.e. in $[T, T+R]$ for $\phi(s)=0, s \in[0, T]$. From equation (2.10) we conclude that

$$
|u(t)|=0 \quad t \in[T, T+R]
$$

since $G>0$ and $\forall \phi \in \mathcal{U}_{D}^{0}$. For the time interval $[0, T]$ we see that

$$
\int_{0}^{T}|u(s)|^{2 \alpha-1} \operatorname{sgn}(u(s)) \phi(s) H(s) \mathrm{d} s=0
$$

This implies that $|u(s)|^{2 \alpha-1} \operatorname{sgn}(u(s)) H(s)$ is in the linear subspace of $L^{2 \alpha}[0, T]$ spanned by the components of $\boldsymbol{F}(T-s) C$. Therefore

$$
|u(s)|^{2 \alpha-1} \operatorname{sgn}(u(s)) H(s)=\lambda \boldsymbol{F}(T-s)
$$

for a constant $\lambda \in \mathbb{R}^{d}$ or

$$
u(s)=\frac{\operatorname{sgn}(\lambda \boldsymbol{F}(T-s))\|\lambda \boldsymbol{F}(T-s)\|^{1 /(2 \alpha-1)}}{H^{1 /(2 \alpha-1)}(s)}
$$

and so the conclusions of theorem 2 follow.

For the general cases, from theorem 2, we have to finally resort to numerical simulations to obtain the solution $u(t)$ of the optimal problem defined in theorem 2. However, for two special cases $\alpha=1$ and $d=1$, the closed form of $u^{*}(t)$ can be obtained as shown in the next two subsections. These special results could give us some basic and fundamental understandings of the behaviour of the optimizer $u^{*}(t)$.

\subsection{Case $\alpha=1$}

For this special case it is readily seen that $u^{*}$ corresponds to $\lambda^{*} \in \mathbb{R}^{d}$ which minimizes

$$
\int_{0}^{T} \frac{\|\boldsymbol{\lambda} \boldsymbol{F}(T-s)\|^{2}}{H(s)} \mathrm{d} s
$$

with the constraint

$$
\int_{0}^{T} F(T-s) \frac{\operatorname{sgn}(\boldsymbol{\lambda} \boldsymbol{F}(T-s))\|\lambda \boldsymbol{F}(T-s)\|}{H(s)} \mathrm{d} s=\boldsymbol{D} .
$$


Define $\boldsymbol{F}(T-s)=\left(v_{1}(s), v_{2}(s), \ldots, v_{d}(s)\right)^{\prime}$ and

$$
W=\left(\int_{0}^{T} \frac{v_{i}(s) v_{j}(s)}{H(s)} \mathrm{d} s\right)_{d \times d} .
$$

If $W^{-1}$ exists, we have

$$
u^{*}(s)=\left\{\begin{array}{cc}
\frac{\boldsymbol{D}^{T}\left(W^{-1}\right)^{T} \boldsymbol{F}(T-s)}{H(s)} & 0 \leqslant s \leqslant T \\
0 & \text { otherwise. }
\end{array}\right.
$$

Basically in [11] Harris and Wolpert carried out a simulated approximation of the results presented in this subsection, i.e. for the case $\alpha=1$.

\subsection{Case $d=1$}

When $d=1$, a straightforward calculation tells us that apart from a constant factor $\boldsymbol{F}(t)$ takes the exponential form $F(t)=\exp (\sigma t)$, and we then have

$u^{*}(t)= \begin{cases}\frac{D}{T} \exp [-\sigma(T-t)] & \text { if } \quad \alpha=1 \\ \frac{D \sigma\left(1-\frac{1}{2 \alpha-1}\right)}{\exp \left[\sigma T\left(1-\frac{1}{2 \alpha-1}\right)\right]-1} \exp \left[-\frac{\sigma}{2 \alpha-1}(T-t)\right] & \text { otherwise }\end{cases}$

for $t \in[0, T]$ and $u(t)=0$ for $t \in[T, T+R]$.

It is interesting to consider the case of Poisson inputs, i.e., $\alpha=1 / 2$ [21]. However, this is the singular case of the results above, i.e., $u^{*}(t)=c_{1} \delta_{0}(t)$ when $\sigma<0$ and $u^{*}(t)=c_{1} \delta_{T}(t)$ for $\sigma>0$, where $c_{1}$ is an appropriate constant. In figure 1 we plot $u^{*}(t)$ versus time $t$, generalized variance at time $t$, namely

$$
\int_{0}^{t} \int_{T}^{T+R}(T+R-v) \exp (2 \sigma(v-s)) \mathrm{d} v\left(u^{*}\right)^{2 \alpha}(s) \mathrm{d} s
$$

(see equation (2.1)) versus $t$ and $\bar{x}(t)=\int_{0}^{t} \exp (\sigma(T-s)) u^{*}(s) \mathrm{d} s$ versus time $t$ for $t \in[0, T]$. For $t \in[T, T+R]$ we have $u(t)=0$.

Furthermore, when $d>1$ and

$$
\boldsymbol{F}(t)=\sum_{i=1}^{d} c_{i+1} \exp \left(\sigma_{i} t\right)
$$

$u^{*}(t)$ can be explicitly calculated as well.

\subsection{Minimizing $\left(\int_{T}^{T+R} \operatorname{tr}[\operatorname{Cov}(x(s))] \mathrm{d} s\right)$}

In the previous subsection we have considered the minimization of the trace of $\operatorname{Cov}\left(\int_{T}^{T+R}\right.$ $\boldsymbol{x}(s) \mathrm{d} s)$. Instead we could of course consider the minimization $\int_{T}^{T+R} \operatorname{tr}[\operatorname{Cov}(\boldsymbol{x}(s))] \mathrm{d} s$, which is another form of generalized variance.

Theorem 3. Let $\boldsymbol{F}(t-s)=\exp ((A-I)(t-s)) \boldsymbol{C}$ for $s \in[0, T]$. Then the unique solution of equations (2.5) and (2.6) $u^{*}(t)$ is given by

$$
u^{*}(s)=\left\{\begin{array}{cl}
\frac{\operatorname{sgn}\left(\boldsymbol{\lambda}^{*} \boldsymbol{F}(T-s)\right)\left\|\boldsymbol{\lambda}^{*} \boldsymbol{F}(T-s)\right\|^{1 /(2 \alpha-1)}}{H^{1 /(2 \alpha-1)}(s)} & t \in[0, T], \\
0 & t \in[T, T+R]
\end{array}\right.
$$



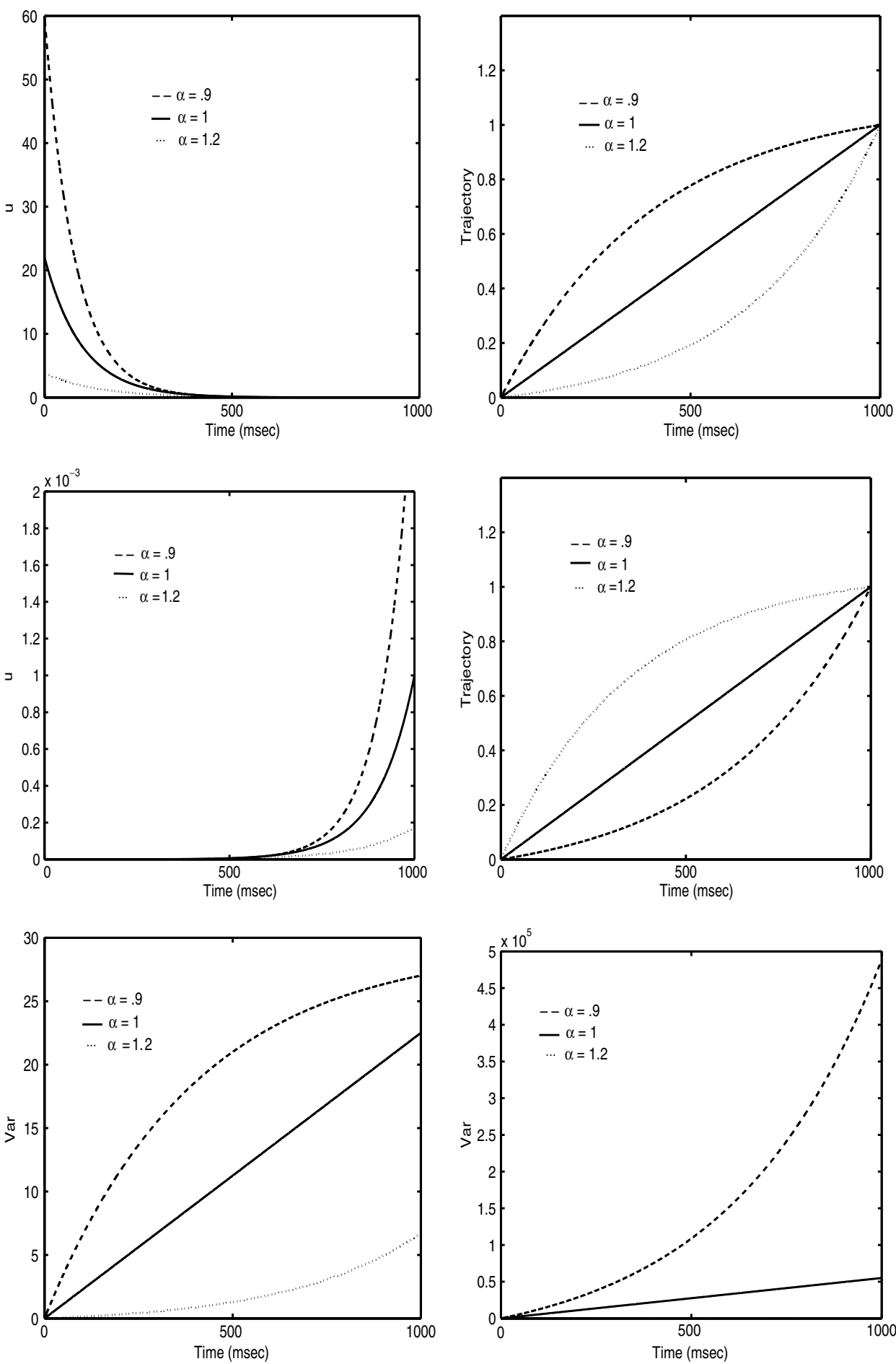

Figure 1. $u$, trajectory and variance versus time. $\sigma=-0.01, R=500, T=1000, d=1$ (upper panels and left bottom panel). $\sigma=0.01, R=500, T=1000, d=1$ (middle panels and right bottom panel). The trajectory is $\bar{x}(t)=\int_{0}^{t} \exp (\sigma(T-s)) u^{*}(s) \mathrm{d} s$. 
where $\boldsymbol{\lambda}^{*} \in \mathbb{R}^{d}$ minimizes

$$
J(\boldsymbol{\lambda})=\left[\int_{0}^{T} \frac{\|\boldsymbol{\lambda} \boldsymbol{F}(T-s)\|^{2 \alpha /(2 \alpha-1)}}{H^{1 /(2 \alpha-1)}(s)} \mathrm{d} s\right]
$$

i.e., $J\left(\boldsymbol{\lambda}^{*}\right)=\inf _{\boldsymbol{\lambda} \in \mathbb{R}^{d}}(J(\boldsymbol{\lambda}))$, with the constraint

$$
\int_{0}^{T} \boldsymbol{F}(T-s) \frac{\operatorname{sgn}(\boldsymbol{\lambda} \boldsymbol{F}(T-s))\|\boldsymbol{\lambda} \boldsymbol{F}(T-s)\|^{1 /(2 \alpha)}}{H^{1 /(2 \alpha-1)}(s)} \mathrm{d} s=\boldsymbol{D}
$$

where $H(s)=\int_{T}^{T+R}\|\boldsymbol{F}(t-s)\|^{2} \mathrm{~d} t ;$ for $t \in[T, T+R], u(t)=0$.

This theorem and its proof are almost the same as theorem 2 except for the different form of $H(s)$.

\section{MVT during movement}

Although results from the original minimum-variance theory fit well with experimental data, one can argue that the results do not concern the equally important issue that the whole trajectory control sometimes is just as important as the post time $(T)$ control. Therefore, we propose the following version of minimum-variance theory: minimization of the variance in a time window $[T-R, T]$ for $T \geqslant R>0$. In principle, our results below can be easily generalized to any subset $A \subset[0, T+R]$ for $R>0$. We can easily prove the following conclusion.

Theorem 4. For $R>0$,

$\mathbf{E} \boldsymbol{Y}^{2}-(\mathbf{E} \boldsymbol{Y})^{2}=2 \int_{T-R}^{T}(T-t) \int_{0}^{t}\|\exp ((A-I)(t-s)) C\|^{2} k^{2} u^{2 \alpha}(s) \mathrm{d} s \mathrm{~d} t$.

We first consider $T>R$ here and deal with the case of $T=R$ in section 3.3. We want to find a real function $u(t)$ which minimizes

$$
2 k^{2} \int_{T-R}^{T}(T-t) \int_{0}^{t}\|\boldsymbol{F}(t-s)\|^{2} u^{2 \alpha}(s) \mathrm{d} s
$$

with the constraint

$$
\int_{0}^{T} \boldsymbol{F}(T-s) u(s) \mathrm{d} s=\boldsymbol{D} \in \mathbb{R}^{d}
$$

where $\boldsymbol{F}(t-s)=\exp ((A-I)(t-s)) \boldsymbol{C}$. As before, after taking routine calculations, we have

$$
\begin{aligned}
I(u)=2 k^{2} \int_{0}^{T-R}|u(s)|^{2 \alpha}\left(\int_{T-R}^{T}(T-t)\|\boldsymbol{F}(t-s)\|^{2} \mathrm{~d} t \mathrm{~d} s\right. \\
\quad+2 k^{2} \int_{T-R}^{T}|u(s)|^{2 \alpha}\left(\int_{T-s}^{T}(T-t)\|\boldsymbol{F}(t-s)\|^{2} \mathrm{~d} t \mathrm{~d} s\right. \\
=2 k^{2} \int_{0}^{T-R}|u(s)|^{2 \alpha} U(s) \mathrm{d} s+2 k^{2} \int_{T-R}^{T}|u(s)|^{2 \alpha} V(s) \mathrm{d} s
\end{aligned}
$$

where

$$
U(s)=\int_{T-R}^{T}(T-t)\|\boldsymbol{F}(t-s)\|^{2} \mathrm{~d} t
$$


for $s \in[0, T-R]$ and

$$
V(s)=\int_{T-s}^{T}(T-t)\|\boldsymbol{F}(t-s)\|^{2} \mathrm{~d} t
$$

for $s \in[T-R, T]$.

The variation problem is reduced to finding the solution of the following equation

$0=\int_{0}^{T-R}|u(s)|^{2 \alpha-1} \operatorname{sgn}(u(s)) \phi(s) U(s) \mathrm{d} s+\int_{T-R}^{T}|u(s)|^{2 \alpha-1} \operatorname{sgn}(u(s)) \phi(s) V(s) \mathrm{d} s$

subject to the condition

$$
\int_{0}^{T} \boldsymbol{F}(T-s) \phi(s) \mathrm{d} s=0
$$

where $\phi \in \mathcal{U}_{D}^{0}$.

Now we consider two special classes of $\phi \in \mathcal{U}_{D}^{0}$.

Class 1. $\phi(s)=0$ for $s \in[T-R, T]$ and $\int_{0}^{T-R} \boldsymbol{F}(T-s) \phi(s) \mathrm{d} s=0$.

Class 2. $\phi(s)=0$ for $s \in[0, T-R]$ and $\int_{T-R}^{T} \boldsymbol{F}(T-s) \phi(s) \mathrm{d} s=0$.

For class 1 we have

$$
|u(s)|^{2 \alpha-1} \operatorname{sgn}(u(s)) U(s)=\boldsymbol{\lambda}^{\prime} \boldsymbol{F}(t-s)
$$

for a vector $\boldsymbol{\lambda} \in \mathbb{R}^{d}$ and $s \in[0, T-R]$.

For class 2, we obtain

$$
|u(s)|^{2 \alpha-1} \operatorname{sgn}(u(s)) V(s)=\boldsymbol{\mu}^{\prime} \boldsymbol{F}(t-s)
$$

for a vector $\mu \in \mathbb{R}^{d}$ and $s \in[T-R, T]$.

Combining the two cases we conclude that

$$
\begin{aligned}
u_{\lambda, \mu}(s)=\frac{\operatorname{sgn}\left(\boldsymbol{\lambda}^{\prime} \boldsymbol{F}(T-s)\right)\left\|\boldsymbol{\lambda}^{\prime} \boldsymbol{F}(T-s)\right\|^{1 /(2 \alpha-1)}}{U^{1 /(2 \alpha-1)}} I_{[0, T-R]} & \\
+\frac{\operatorname{sgn}\left(\boldsymbol{\mu}^{\prime} \boldsymbol{F}(T-s)\right)\left\|\boldsymbol{\mu}^{\prime} \boldsymbol{F}(T-s)\right\|^{1 /(2 \alpha-1)}}{V^{1 /(2 \alpha-1)}} & I_{[T-R, T]}
\end{aligned}
$$

where $I$ is the indicator function, i.e. $I_{A}(x)=1$ if $x \in A$ for a set $A$ and 0 otherwise. Hence the original problem is now reduced to the following much simpler problem: to find two vectors $\lambda^{*}$ and $\mu^{*}$ to minimize the objective function

$K(\boldsymbol{\lambda}, \boldsymbol{\mu})=\int_{0}^{T-R} \frac{\left\|\boldsymbol{\lambda}^{\prime} \boldsymbol{F}(T-s)\right\|^{2 \alpha /(2 \alpha-1)}}{U^{1 /(2 \alpha-1)}} \mathrm{d} s+\int_{T-R}^{T} \frac{\left\|\boldsymbol{\mu}^{\prime} \boldsymbol{F}(T-s)\right\|^{2 \alpha /(2 \alpha-1)}}{V^{1 /(2 \alpha-1)}} \mathrm{d} s$

such that $K\left(\boldsymbol{\lambda}^{*}, \boldsymbol{\mu}^{*}\right)=\min _{\lambda, \mu \in \mathbb{R}^{d}} K(\boldsymbol{\lambda}, \mu)$, with the constraint

$$
\begin{aligned}
\int_{0}^{T-R} \boldsymbol{F}(T-s) & \frac{\operatorname{sgn}\left(\boldsymbol{\lambda}^{\prime} \boldsymbol{F}(T-s)\right)\left\|\boldsymbol{\lambda}^{\prime} \boldsymbol{F}(T-s)\right\|^{1 /(2 \alpha-1)}}{U^{1 /(2 \alpha-1)}} \mathrm{d} s \\
& +\int_{T-R}^{T} \boldsymbol{F}(T-s) \frac{\operatorname{sgn}\left(\boldsymbol{\mu}^{\prime} \boldsymbol{F}(T-s)\right)\left\|\boldsymbol{\mu}^{\prime} \boldsymbol{F}(T-s)\right\|^{1 /(2 \alpha-1)}}{V^{1 /(2 \alpha-1)}} \mathrm{d} s=\boldsymbol{D} .
\end{aligned}
$$

For general cases we have to resort to numerical solutions, but for two special cases: $\alpha=1$ and $d=1$, we can obtain further analytical solutions. 


\subsection{Case $\alpha=1$}

In this case equations (3.5) and (3.6) become

$$
K(\boldsymbol{\lambda}, \boldsymbol{\mu})=\int_{0}^{T-R} \frac{\left[\boldsymbol{\lambda}^{\prime} \boldsymbol{F}(T-s)\right]^{2}}{U} \mathrm{~d} s+\int_{T-R}^{T} \frac{\left[\boldsymbol{\mu}^{\prime} \boldsymbol{F}(T-s)\right]^{2}}{V} \mathrm{~d} s
$$

and

$$
\int_{0}^{T-R} \boldsymbol{F}(T-s) \frac{\left(\boldsymbol{\lambda}^{\prime} \boldsymbol{F}(T-s)\right)}{U} \mathrm{~d} s+\int_{T-R}^{T} \boldsymbol{F}(T-s) \frac{\left(\boldsymbol{\mu}^{\prime} \boldsymbol{F}(T-s)\right)}{V} \mathrm{~d} s=\boldsymbol{D} .
$$

Let us denote $\boldsymbol{F}(T-s)=\left(v_{1}(s), \ldots, v_{d}(s)\right)^{\prime}$ and

$$
\left\{\begin{array}{l}
X=\left(\int_{0}^{T-R} \frac{v_{i}(s) v_{j}(s)}{U(s)} \mathrm{d} s\right)_{d \times d} \\
Y=\left(\int_{T-R}^{T} \frac{v_{i}(s) v_{j}(s)}{V(s)} \mathrm{d} s\right)_{d \times d} .
\end{array}\right.
$$

The original problem can be stated as to minimize

$$
\lambda^{\prime} X \lambda+\mu^{\prime} Y \mu
$$

with the constraint $X \boldsymbol{\lambda}+Y \boldsymbol{\mu}=\boldsymbol{D}$. If we further assume that $X^{-1}$ exists, we get

$$
\left\{\begin{array}{l}
\boldsymbol{\mu}^{*}=\left(Y X^{-1} Y+Y\right)^{-1} Y X^{-1} \boldsymbol{D} \\
\boldsymbol{\lambda}^{*}=X^{-1} \boldsymbol{D}-X^{-1} Y \boldsymbol{\mu}
\end{array}\right.
$$

which yields

$$
\begin{aligned}
& u^{*}=\frac{\left(X^{-1} \boldsymbol{D}-X^{-1} Y \boldsymbol{\mu}^{*}\right) \boldsymbol{F}(T-s)}{U(s)} I_{[0, T-R]} \\
& +\frac{\left(\left(Y X^{-1} Y+Y\right)^{-1} Y X^{-1} \boldsymbol{D}\right) \boldsymbol{F}(T-s)}{V(s)} I_{[T-R, T]} .
\end{aligned}
$$

3.2. Case $d=1$

Without loss of generality we can assume that $F(t-s)=\exp (\sigma(t-s))$. Now $u_{\lambda, \mu}$ is

$$
\begin{aligned}
u_{\lambda, \mu}=\operatorname{sgn}(\lambda)|\lambda|^{1 /(2 \alpha-1)}\left(\frac{F(T-s)}{U(s)}\right)^{1 /(2 \alpha-1)} I_{[0, T-R]} \\
+\operatorname{sgn}(\mu)|\mu|^{1 /(2 \alpha-1)}\left(\frac{F(T-s)}{V(s)}\right)^{1 /(2 \alpha-1)} I_{[T-R, T]}
\end{aligned}
$$

and the objective function (3.5) and the constraint (3.6), respectively, become

$$
K_{\lambda, \mu}=\int_{0}^{T-R} \frac{|\lambda|^{2 \alpha /(2 \alpha-1)}(F(T-s))^{2 \alpha /(2 \alpha-1)}}{U^{1 /(2 \alpha-1)}(s)} \mathrm{d} s+\int_{T-R}^{T} \frac{|\mu|^{2 \alpha /(2 \alpha-1)}(F(T-s))^{2 \alpha /(2 \alpha-1)}}{V^{1 /(2 \alpha-1)}(s)} \mathrm{d} s
$$

and

$$
\begin{aligned}
D=\operatorname{sgn}(\lambda)|\lambda|^{1 /(2 \alpha-1)} \int_{0}^{T-R} \frac{F^{2 \alpha /(2 \alpha-1)}(T-s)}{U^{1 /(2 \alpha-1)}(s)} \mathrm{d} s \\
+\operatorname{sgn}(\mu)|\mu|^{1 /(2 \alpha-1)} \int_{T-R}^{T} \frac{F^{2 \alpha /(2 \alpha-1)}(T-s)}{V^{1 /(2 \alpha-1)}(s)} \mathrm{d} s .
\end{aligned}
$$


We further introduce

$$
\begin{aligned}
& \hat{\lambda}=\operatorname{sgn}(\lambda)|\lambda|^{1 /(2 \alpha-1)} \quad \hat{\mu}=\operatorname{sgn}(\mu)|\mu|^{1 /(2 \alpha-1)} \\
& a=\int_{0}^{T-R} \frac{F^{2 \alpha /(2 \alpha-1)}(T-s)}{U^{1 /(2 \alpha-1)}} \mathrm{d} s \\
& =\left[\frac{4 \sigma^{2}}{1-(2 \sigma R+1) \exp (-2 \sigma R)}\right]^{1 /(2 \alpha-1)} \int_{0}^{T-R} \exp \left(\frac{2 \sigma(T-s)(\alpha-1)}{2 \alpha-1}\right) \mathrm{d} s \\
& \int\left[\frac{4 \sigma^{2}}{1-(2 \sigma R+1) \exp (-2 \sigma R)}\right]^{\frac{1}{(2 \alpha-1)}}\left[\exp \left(\frac{2 \sigma T(\alpha-1)}{2 \alpha-1}\right)\right. \\
& = \begin{cases}\left.-\exp \left(\frac{2 \sigma R(\alpha-1)}{2 \alpha-1}\right)\right] \frac{2 \alpha-1}{2(\alpha-1) \sigma} & \text { if } \quad \alpha \neq 1 \\
{\left[\frac{4 \sigma^{2}}{1-(2 \sigma R+1) \exp (-2 \sigma R)}\right](T-R)} & \text { if } \quad \alpha=1\end{cases}
\end{aligned}
$$

and

$$
\begin{aligned}
b & =\int_{T-R}^{T} \frac{F^{2 \alpha /(2 \alpha-1)}(T-s)}{V^{1 /(2 \alpha-1)} \mathrm{d} s} \\
& =\int_{T-R}^{T}\left[\frac{4 \sigma^{2}}{1-(2 \sigma s+1) \exp (-2 \sigma s)}\right]^{1 /(2 \alpha-1)} \exp \left(\frac{2 \sigma(\alpha-1)(T-s)}{2 \alpha-1}\right) \mathrm{d} s \\
& = \begin{cases}\int_{T-R}^{T}\left[\frac{4 \sigma^{2}}{1-(2 \sigma s+1) \exp (-2 \sigma s)}\right]^{1 /(2 \alpha-1)} \exp \left(\frac{2 \sigma(\alpha-1)(T-s)}{2 \alpha-1}\right) \mathrm{d} s & \text { if } \quad \alpha \neq 1 \\
\int_{T-R}^{T}\left[\frac{4 \sigma^{2}}{1-(2 \sigma s+1) \exp (-2 \sigma s)}\right] \mathrm{d} s & \text { if } \quad \alpha=1 .\end{cases}
\end{aligned}
$$

With the given $a$ and $b$, the original variation problem is now simplified to minimize

$$
|\hat{\lambda}|^{2 \alpha} a+|\hat{\mu}|^{2 \alpha} b
$$

with constraint

$$
\hat{\lambda} a+\hat{\mu} b=D .
$$

Using the Lagrangian multiplier, one obtains

$$
\left\{\begin{array}{l}
\hat{\lambda}=\frac{D}{a^{2 \alpha /(2 \alpha-1)}+b^{2 \alpha /(2 \alpha-1)}} a^{1 /(2 \alpha-1)} \\
\hat{\mu}=\frac{D}{a^{2 \alpha /(2 \alpha-1)}+b^{2 \alpha /(2 \alpha-1)}} b^{1 /(2 \alpha-1)} .
\end{array}\right.
$$

Therefore,

$$
\left\{\begin{array}{l}
\lambda=\frac{\operatorname{sgn}(D)|D|^{2 \alpha-1}}{\left(a^{2 \alpha /(2 \alpha-1)}+b^{2 \alpha /(2 \alpha-1)}\right)^{2 \alpha-1}} a \\
\mu=\frac{\operatorname{sgn}(D)|D|^{2 \alpha-1}}{\left(a^{2 \alpha /(2 \alpha-1)}+b^{2 \alpha /(2 \alpha-1)}\right)^{2 \alpha-1}} b .
\end{array}\right.
$$

Noting that

$$
\left(\frac{F(T-s)}{U(s)}\right)^{1 /(2 \alpha-1)}=\left[\frac{4 \sigma^{2}}{1-(2 \sigma R+1) \exp (-2 \sigma R))}\right]^{1 /(2 \alpha-1)} \exp \left(\frac{-\sigma(T-s)}{2 \alpha-1}\right)
$$


and

$$
\left(\frac{F(T-s)}{V(s)}\right)^{1 /(2 \alpha-1)}=\left[\frac{4 \sigma^{2}}{1-(2 \sigma s+1) \exp (-2 \sigma s)}\right]^{1 /(2 \alpha-1)} \exp \left(\frac{-\sigma(T-s)}{2 \alpha-1}\right)
$$

we could see that the general solution of the original problem is composed of two exponential functions. The coefficient of the first exponential function is independent of time while the second is not. In particular, when $\alpha=1$,

and

$$
\left(\frac{F(T-s)}{U(s)}\right)=\left[\frac{4 \sigma^{2}}{1-(2 \sigma R+1) \exp (-2 \sigma R)}\right] \exp (-\sigma(T-s))
$$

$$
\left(\frac{F(T-s)}{V(s)}\right)=\left[\frac{4 \sigma^{2}}{1-(2 \sigma s+1) \exp (-2 \sigma s)}\right] \exp (-\sigma(T-s))
$$

In summary, $u^{*}$ is given by

$$
\begin{aligned}
u^{*}(s)=\frac{D}{a^{2 \alpha /(2 \alpha-1)}+b^{2 \alpha /(2 \alpha-1)}} a^{1 /(2 \alpha-1)}\left(\frac{F(T-s)}{U(s)}\right)^{1 /(2 \alpha-1)} I_{[0, T-R]} \\
+\frac{D}{a^{2 \alpha /(2 \alpha-1)}+b^{2 \alpha /(2 \alpha-1)}} b^{1 /(2 \alpha-1)}\left(\frac{F(T-s)}{V(s)}\right)^{1 /(2 \alpha-1)} I_{[T-R, T]} .
\end{aligned}
$$

In figures 2 and 3 numerical results of $u^{*}(t)$, general variance at time $t$ and $\bar{x}(t)$ versus time $t$ are shown for $\sigma>0$ and $\sigma<0$. Basically $u^{*}(t)$ is composed of two pieces of exponential function. If minimizing the variance is the principle, then we see that the variance attains its minimum when $\sigma>0$ and $\alpha=1.2$. It is illuminating to point out that the variance in the previous section is smaller than the variance here when $\sigma<0$, but is larger when $\sigma>0$. As in the previous section, when $\alpha \rightarrow 1 / 2$ the solution tends to $c_{1} \delta_{0}$ or $c_{1} \delta_{T}$, where $c_{1}$ is an appropriate constant.

Finally as in the previous section, we can also consider the minimization of $\left(\int_{T-R}^{T} \operatorname{tr}[\operatorname{Cov}(\boldsymbol{x}(s)) \mathrm{d} s]\right)$. Since the results are similar we leave them to the reader as an exercise.

\subsection{Whole range control}

If minimization of the variance is the purpose of a biological system, one would argue that we should consider the case of $R=T$, i.e. the case of whole range control. Unfortunately, the solution is degenerate. In fact from equation (3.1) we see that $V(0)=0$, and so the solution of equation (3.3) is not properly defined. A natural treatment is to define the solution $u^{*}(t)$ at $t=0$ as the limit of $t \rightarrow 0$. Let us consider a specific case: $d=1$. From equation (3.13) we see that

$$
\lim _{s \rightarrow 0} u^{*}(s)=\lim _{s \rightarrow 0} \frac{D}{b}\left(\frac{F(T-s)}{V(s)}\right)^{1 /(2 \alpha-1)} I_{[0, T]} .
$$

Note that $b \sim s^{(2 \alpha-3) /(2 \alpha-1)}$ and $(F(T-s) / V(s))^{1 /(2 \alpha-1)} \sim s^{-2 /(2 \alpha-1)}$, we conclude that $u^{*}(s) \sim s^{-1}$. Therefore the solution approaches infinity as $t \rightarrow 0$. Although the solution does not exist for the whole range control, we can define the solution within a time window $[T-R, T]$ for $R \sim T$. As one might expect, in this case the variance is considerably reduced. Let us write the general variance at time $t$ as $\operatorname{var}(t)$; in figure 4, we plot $u^{*}(t)$ and $\operatorname{var}(t)$ versus $t$. It is easily seen that $\operatorname{var}(t)$ is a decreasing function of $R$. Combining figures $1-4$ together, we see that when $R=999$, the variance attains its minimal value (not surprising). The true trajectory $E x(t)$ (see next section) is quite similar (not shown) for $R=900,990,999$. 

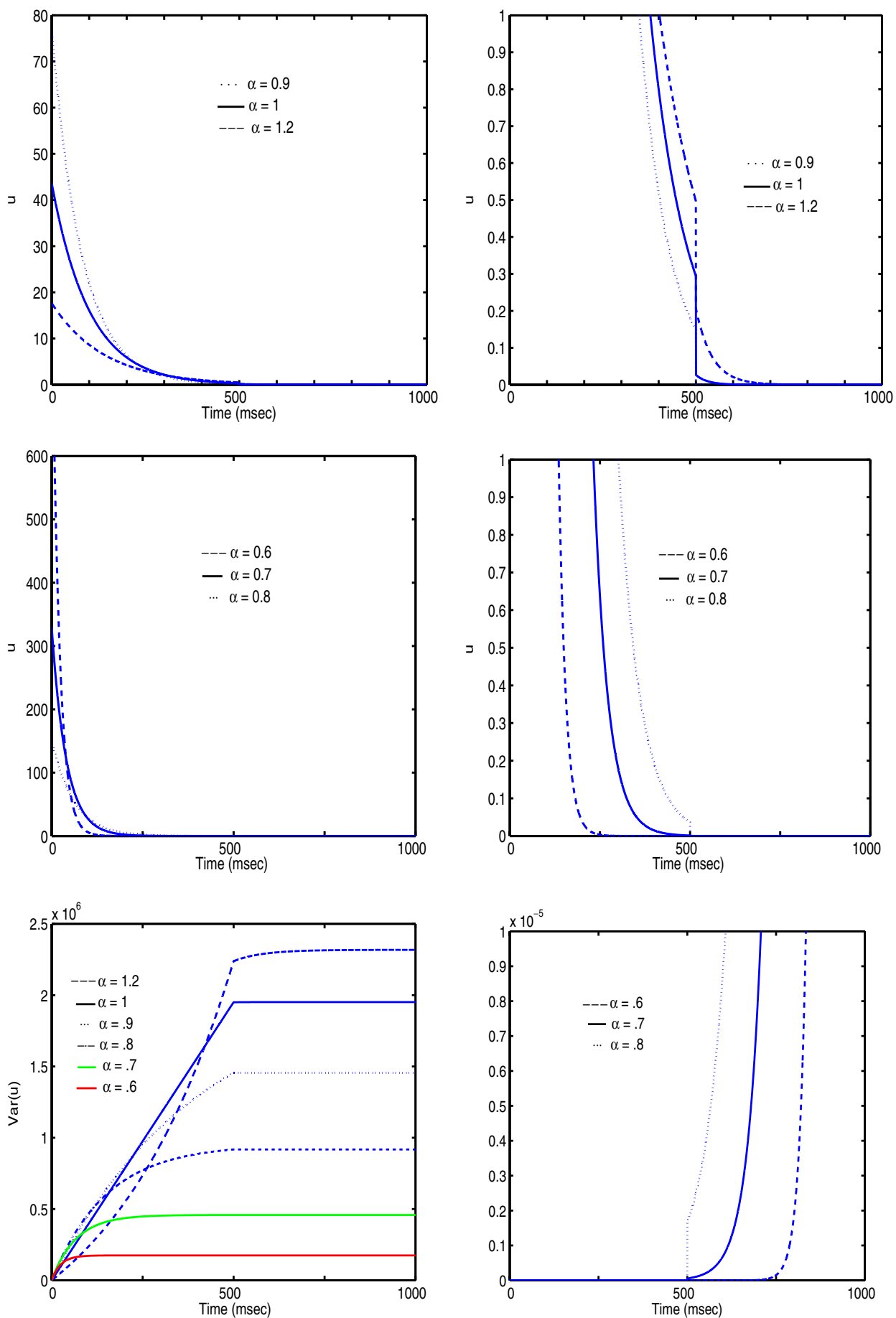

Figure 2. $\sigma=-0.01, R=500, T=1000, D=1 . \quad \operatorname{var}(t)=2 \int_{0}^{\min (t, T-R)}\left|u^{*}(s)\right|^{2 \alpha} U(s) \mathrm{d} s+$ $2 \int_{\min (t, T-R)}^{\min (t, T)}\left|u^{*}(s)\right|^{2 \alpha} V(s) \mathrm{d} s$ versus time $t$. The trajectory is $\bar{x}(t)=\int_{0}^{t} \exp (\sigma(T-s)) u^{*}(s) \mathrm{d} s$. Upper and middle panels are for $\alpha=0.6,0.7,0.8,0.9,1.0,1.2$, the right is a blow-up of the left. The bottom panels show var and trajectory. 

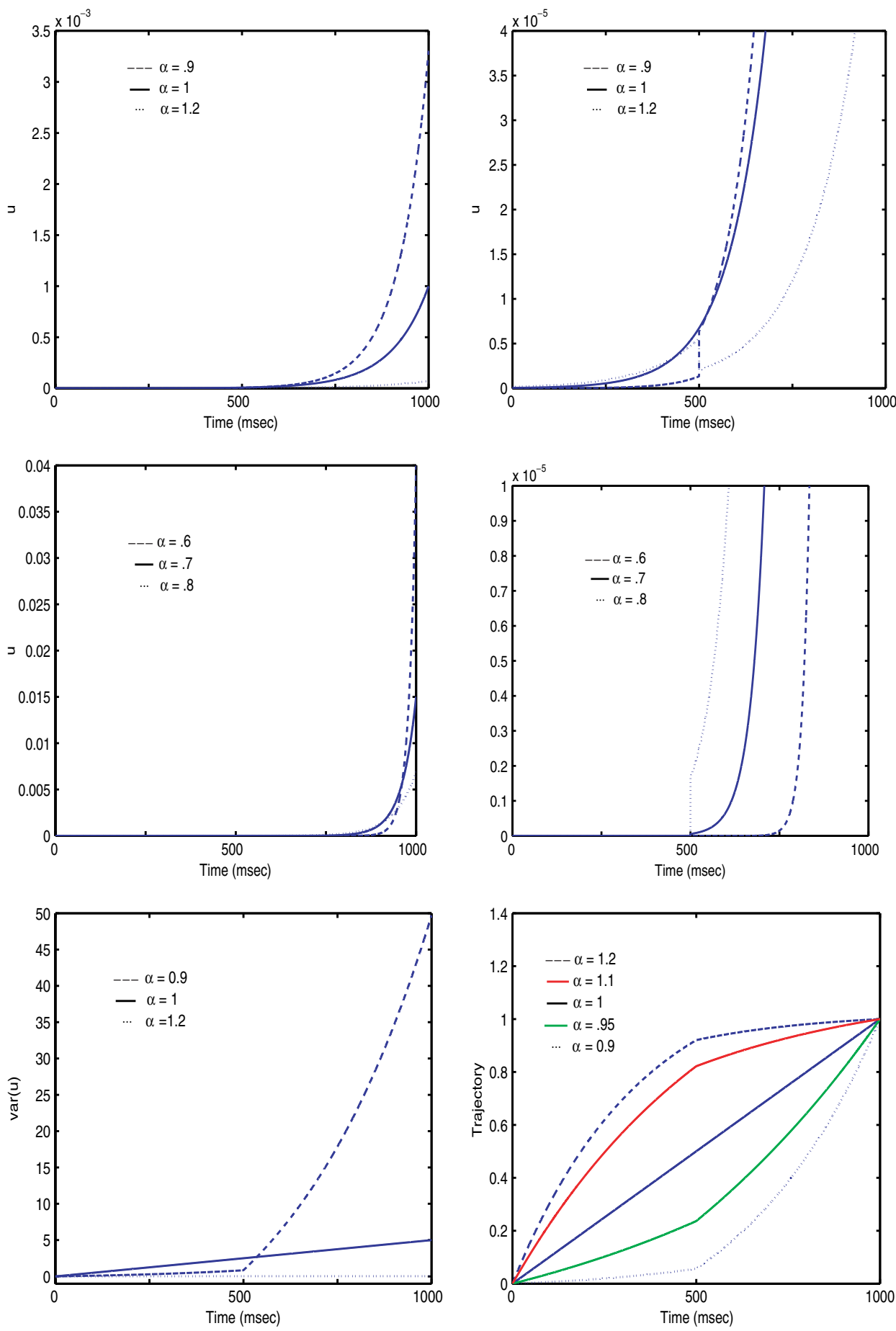

Figure 3. $\sigma=0.01, R=500, T=1000, D=1 . \quad \operatorname{var}(t)=2 \int_{0}^{\min (t, T-R)}\left|u^{*}(s)\right|^{2 \alpha} U(s) \mathrm{d} s+$ $2 \int_{\min (t, T-R)}^{\min (t, T)}\left|u^{*}(s)\right|^{2 \alpha} V(s) \mathrm{d} s$ versus time $t$. The trajectory is $\bar{x}(t)=\int_{0}^{t} \exp \left(\sigma(T-s) u^{*}(s) \mathrm{d} s\right.$. Upper and middle panels are for $\alpha=0.6,0.7,0.8,0.9,1.0,1.2$, the right is a blow-up of the left. The bottom panels show var and trajectory. 

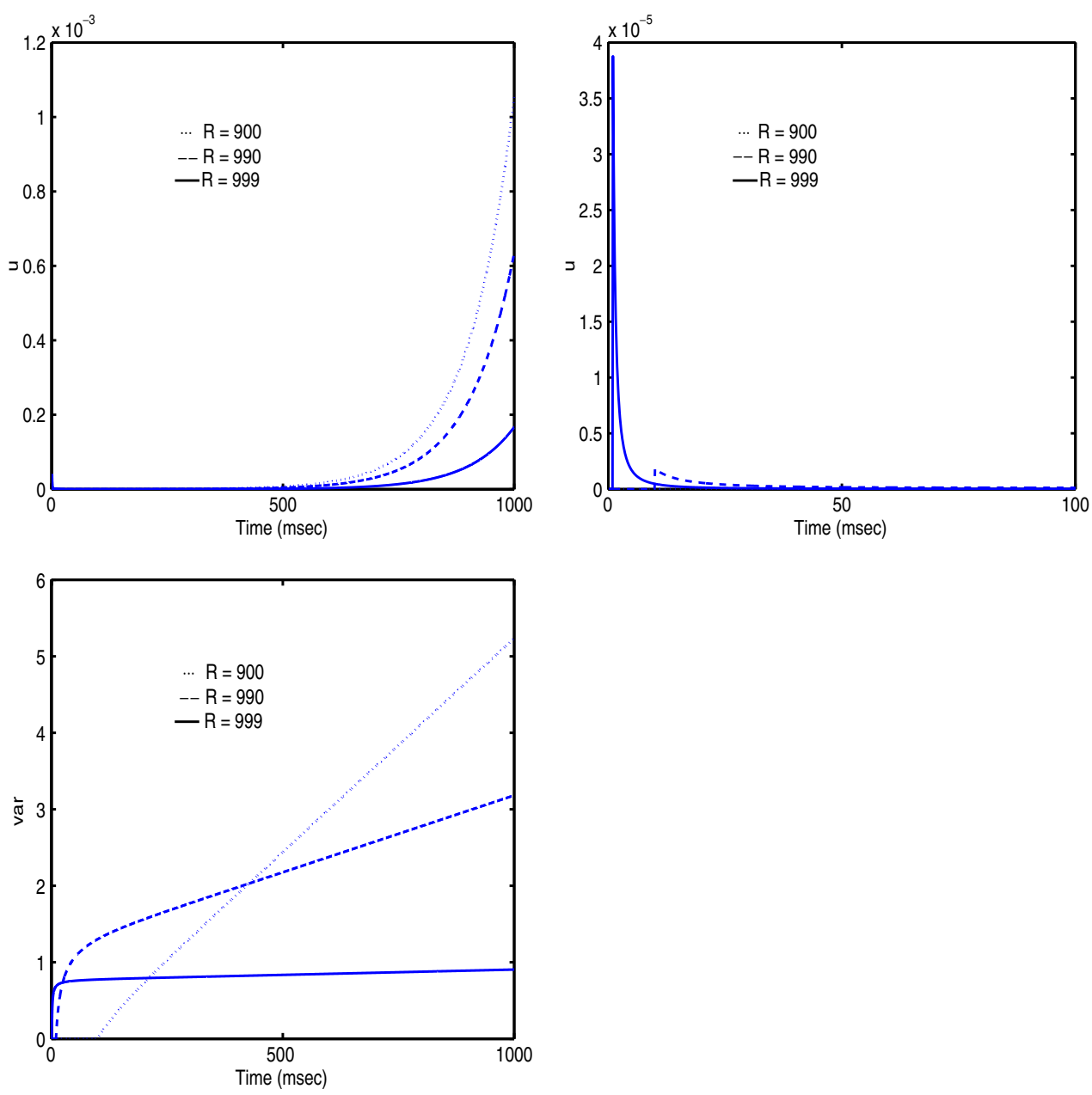

Figure 4. $\sigma=0.01, R=900,990,999, T=1000, D=1, \alpha=1 . u^{*}(t)$ versus time $t$ (upper panels), $\operatorname{var}(t)=2 \int_{0}^{\min (t, T-R)}\left|u^{*}(s)\right|^{2 \alpha} U(s) \mathrm{d} s+2 \int_{\min (t, T-R)}^{\min (t, T)}\left|u^{*}(s)\right|^{2 \alpha} V(s) \mathrm{d} s$ versus time $t$ (bottom panel).

\section{Discussion}

We presented a theoretical analysis for the minimum-variance theory of post and during movement. Basically for post-movement optimization, it is required that the solution (input signal) vanish after movement, for during movement optimization the solution consists of two exponential functions when $d=1$. Most interestingly, we have numerically found that the variance is considerably reduced for the minimum-variance theory of during movement, in comparison with the minimum-variance theory of post movement. Our results presented here would shed new light on the optimal control theory, in particular on biological control theory (for example, see [3, 12]).

Although the minimum-variance theory is interesting, we have to point out a few key shortcomings of the theory. First of all, it is generally believed that neuron signals take the form of a Poisson process, rather than a general renewal process [6]. For a Poisson process, 

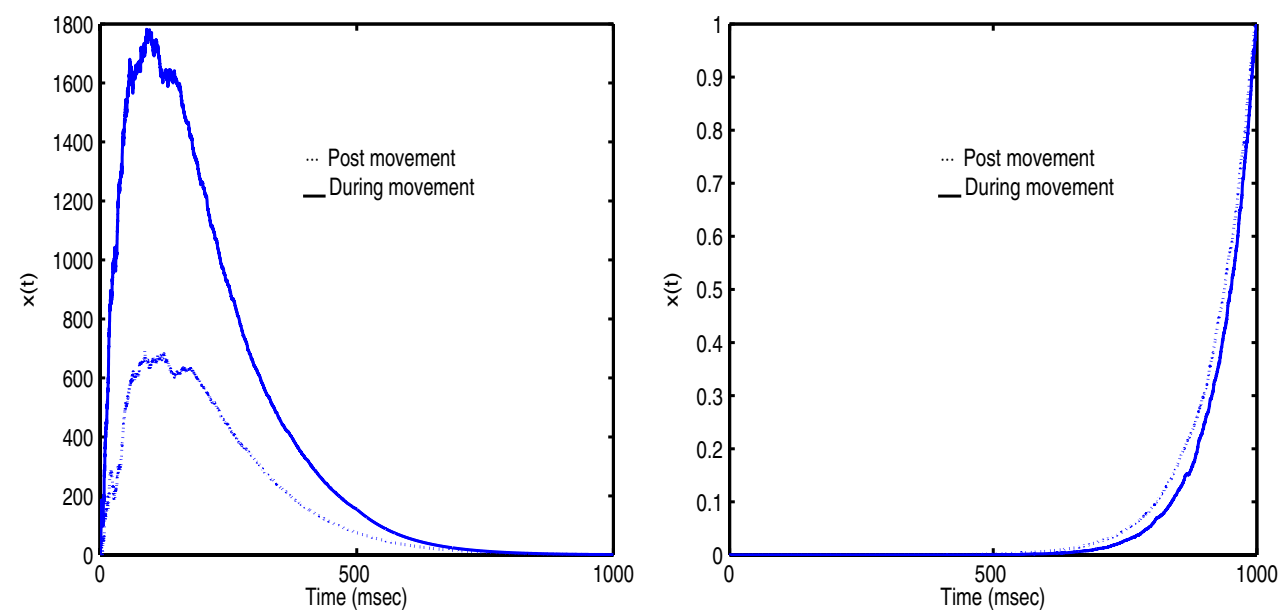

Figure 5. $x(t)$ versus time $t$ with parameters $\sigma=-0.01, R=500, T=1000, D=1, \alpha=1$ (left); $\sigma=0.01, R=500, T=1000, D=1, \alpha=0.9$ (right).

we have $\alpha=1 / 2$ and we know that the solution of the minimum-variance theory is degenerate, for both during and post movement. When $\alpha=1$, the case considered in [11], we have a coefficient of variation equal to $u^{\alpha}(t)$, which is too high for neuron signals [4]. Nevertheless, the issue of exactly which forms of signals are formed by about 200000 alpha motor neurons remains controversial and further study is required [23].

It would be interesting to test the theory with a more realistic neuron signal: to replace $u(t)$ by a sequence of Poisson processes [6]. As aforementioned, Poisson processes are not suitable for the theory. However, it has recently been realized that input Poisson processes are correlated $[18,4]$. With correlated inputs, the input form would be significantly different from the form considered in the current paper.

In statistical theory, to minimize the variance of an estimator is a basic approach. Based upon it, many theories such as the Fisher's information function have been developed. One of the most appealing features of the minimum-variance theory is probably the close relationship with statistical theory. To further pursue the topic, for example, establishing a relationship between the Fisher information and the minimum-variance theory, is obviously a challenging issue.

All results obtained here rely on the linear system defined in section 1. Certainly in reality a biological system is highly nonlinear. How to generalize our results here to a nonlinear system is an intriguing topic. In fact, for all problems considered in the current paper, we have first fixed a time $T$. Certainly a more reasonable approach should define a stopping time

$$
T=\inf \{t: \boldsymbol{x}(t)=\boldsymbol{D}\}
$$

where $\boldsymbol{x}(t)$ is given by equation (1.3) and we seek to find a solution to minimize the variance in a time window, say $[T / 2, T]$. In terms of this approach we might get rid of the singularity when inputs are Poisson processes since the optimization problem is then no longer linear, i.e., only dependent on $|u(t)|$. The difficulty of such an approach lies in the fact that it is not easy to find the distribution of $T$, despite many years of research endeavour [5]. Nevertheless, different approximation methods have been developed [4], and we will publish them in future publications. In fact, the introduction of stopping time becomes essential when $\sigma<0$. Looking at figures 1-3 one must be curious to observe that the variance is so large when $\sigma<0$ and would want to know the source of it. In figure 5, we plot the actual trajectory of $x(t)$ 
(note that in figures $1-3 \bar{x}(t)$ is plotted) for $\sigma<0$ and $\sigma>0$. It is easily seen that $x(t)>1$ when $t \in[0,1000]$ and $\sigma<0$. From the uniqueness of the solution (see theorems in previous sections), we see that there is no solution when $\sigma<0$ if we further require that $x(t)<1$ for $t \in[0,1000)$. Within the framework of the stochastic control theory, i.e. introducing the stopping time, we can resolve the problem.

\section{Acknowledgment}

We would like to thank one of the referees for his/her critical comments on an early version of the paper. This work was partially supported by a grant from EPSRC(GR/R54569) and the Royal Society (JF).

\section{References}

[1] Albright T D, Jessell T M, Kandel E R and Posner M I 2000 Neural science: a century of progress and the mysteries that remain Cell $100 \mathrm{~s} 1-\mathrm{s} 55$

[2] de Vries G and Sherman A 2000 Channel sharing in pancreatic beta-cells revisited: enhancement of emergent bursting by noise J. Theor. Biol. 207 513-30

[3] Doya K, Kimura H and Kawato M 2001 Neural mechanisms of learning and control IEEE Control Syst. Mag. 21 42-54

[4] Feng J F 2001 Is the integrate-and-fire model good enough? - a review Neural Netw. 14 955-75

[5] Feng J F 2002 Frontiers in Computational Neuroscience ed J F Feng (Boca Raton, FL: CRC Press)

[6] Feng J F, Tartaglia G G and Tirozzi B 2001 A note on minimum-variance theory and beyond at press

[7] Gammaitoni L, Hänggi P, Jung P and Marchesoni F 1998 Stochastic resonance Rev. Mod. Phys. 70 224-87

[8] Gerstner W, Kreiter A K, Markram H and Herz A V M 1997 Neural codes: firing rates and beyond Proc. Natl Acad. Sci. USA 94 12740-1

[9] Hopfield J J and Brody C D 2000 What is a moment? 'Cortical' sensory integration over a brief interval Proc. Natl Acad. Sci. USA 97 13919-24

[10] Hopfield J J and Brody C D 2001 What is a moment? Transient synchrony as a collective mechanism for spatiotemporal integration Proc. Natl Acad. Sci. USA 98 1282-7

[11] Harris C M and Wolpert D M 1998 Signal-dependent noise determines motor planning Nature 394 780-4

[12] Kawato M 1999 Internal models for motor control and trajectory planning Curr. Opin. Neurobiol. 9 718-27

[13] Koch C 1999 Biophysics of Computation (Oxford: Oxford University Press)

[14] Kushner H J 1999 Consistency issues for numerical methods for variance control, with applicatinos to optimization in finance IEEE Trans. Automat. Contr. 44 2283-96

[15] Karlin S and Taylor H M 1975 A First Course in Stochastic Processes 2nd edn (Boston, MA: Academic)

[16] Oksendal B 1989 Stochastic Differential Equations 2nd edn (Berlin: Springer)

[17] Ricciardi L M and Sato S 1990 Diffusion process and first-passage-times problems Lectures in Applied Mathematics and Informatics ed L M Ricciardi (Manchester: Manchester University Press)

[18] Salinas E and Sejnowski T 2001 Correlated neuronal activity and the flow of neural information Nature Rev. Neurosci. 2 539-50

[19] Sejnowski T J 1998 Making smooth moves Nature 394 725-6

[20] Shadlen M N and Newsome W T 1994 Noise, neural codes and cortical organization Curr. Opin. Neurobiol. 4 569-79

[21] Tuckwell H C 1988 Introduction to Theoretical Neurobiology vol 2 (Cambridge: Cambridge University Press)

[22] Wolpert D M 2001 Private communication

[23] Wolpert D M and Ghahramani Z 2000 Computational principles of movement neuroscience Nat. Neurosci. 3 $1212-7$ 